\title{
REVIEW
}

\section{COVID-19 PANDEMIC AND THE MENTAL HEALTH OF ELDERLY}

Turkish Journal of Geriatrics

DOI: $10.31086 /$ tigeri.2020.165

2020; 23(3): 291-298

_ Özlem Erden AKi ${ }^{1}$

CORRESPONDANCE

Özlem Erden AK

Hacettepe University, School of Medicine

Department of Psychiatry, Ankara, Turkey

Phone: +903123051440

e-mail: ozlemerdenaki@yahoo.com

Received: Jun 30, 2020

Accepted: Aug 07, 2020

${ }^{1}$ Hacettepe University, School of Medicine, Department of Psychiatry, Ankara, Turkey

\section{A}

The new type of corona virus (SARS-Cov 2) pandemic, which broke out in Wuhan province of China in December 2019, has spread rapidly in a short time, affected and is still affecting millions of people all over the world. Numerous measures have been taken to prevent the spread of the outbreak, in particular "maintaining social distance". Elderly individuals are vulnerable to COVID-19 due to the aging of their immune systems and concomitant chronic diseases; morbidity and mortality are rather high in this age group, so prevention measures are even of greater importance in older individuals. However, both the pandemic and the infection control measures taken for the pandemic negatively affect the mental health of elderly individuals as well as their physical health. In this review study, it was aimed to examine briefly the effects of pandemics on mental health of the society, to review the studies investigating the effects of outbreaks on the mental health of the elderly, and to make inferences for the future.

Keywords: Coronavirus; Pandemics; Aged; Mental health 


\section{INTRODUCTION}

Started in Wuhan province of China in December 2019, the new type of Coronavirus (SARS-Cov 2) outbreak has spread rapidly all over the world and affected thousands of people in almost every country. Many epidemics caused by viruses (like SARS and MERS) have been around seen since the beginning of the twenty-first century, but among them, COVID-19 seems to have spread at the highest speed and caused enormous number of deaths (1). The World Health Organization (WHO) declared the new type of Coronavirus outbreak as a pandemic on 11 March 2020. Measures have been taken by authorities in almost all countries of the world in order to prevent the spread of SARS-Cov-2 infection, which has a higher range of contagiousness compared to previous Coronavirus infections (2). In addition to the fundamental measures such as the use of face masks in public areas, following general basic hygiene rules, maintaining a distance of at least 2 meters from others called as "social distancing", other measures such as stopping production in non-essential business sectors, quarantine and curfews have also been implemented in some countries.

Although the SARS-Cov-2 virus affects individuals of all ages, older individuals have been found to be more vulnerable and at higher risk of infection due to their declining immune systems and concomitant chronic diseases. In older adults, the illness follows a more severe and prolonged course; secondary bacterial infection and respiratory failure is reported to develop more frequently and intensive care treatment is needed with additional pharmacological treatments (3). The elderly people, often fragile and with a number of concomitant chronic diseases, should be closely monitored during and after the treatment, and additional measures should be implemented for rehabilitation. However, in addition to many physical problems, those elderly adults are also deprived of the help and social support of their caregivers due to the social distancing measures both at home and in the hospital, and become socially isolated (4).

The high mortality and morbidity associated with the disease makes the protection measures more crucial in the elderly population. Though the epidemic broke out a couple of months ago, health authorities such as the World Health Organization have developed a guideline for the elderly and the staff in nursing homes, but they have not developed any guidelines for older people in the community, which comprises the majority of the elderly population (5). With the circular of the Ministry of Internal Affairs issued in our country on 21 March 2020, a curfew for the people aged 65 and over and those having additional chronic diseases were implemented. In other countries, such a restrictive curfew decision was not taken for the elderly, but older people were recommended not to enter the crowds as much as possible to maintain social distance. Although such control measures have a positive effect on disease prevention, they also make it difficult for older individuals to implement their essential daily activities such as shopping, bank transactions, and health care services, leading them to stay away from social relations, which is very important especially in the old age, and cause them to experience some kind of social isolation. Curfews have been progressively relaxed and eventually lifted in some areas for the general public; however, as the risk of transmission of the virus continues, the older adults are recommended to continue their protection and isolation measures. Therefore, the elderly will likely continue their lives with restrictions for a longer period of time. As a result, both the disease process and prevention measures appear to cause elderly individuals to experience additional psychological difficulties.

Natural disasters, epidemics and preventive measures like keeping social distance have critical effects on the mental health of individuals both in the short and long term. From the beginning of the twenty-first century, numerous outbreaks caused by different viruses have been thought to affect the psychology of the whole society and also the groups 
affected by the epidemic at differing levels (6); and numerous studies have been carried out on the psychological effects of epidemics both during and after the outbreak. The data obtained from previous epidemic experiences are quite valuable for the road mapping of the current and future outbreaks. In this review, it was aimed to investigate briefly the effects of outbreaks on the mental health of society as a whole, and to review the studies examining the effects of epidemics on elderly individuals' mental health and to make inferences for the future.

\section{Effects of epidemics on mental health}

The research focusing on the effects of epidemic diseases on mental health has generally been carried out on those who are primarily affected by the disease, healthcare professionals or on the individuals who survive the disease. Post-traumatic stress symptoms, anxiety and depression symptoms, anger and stigmatization were investigated in those groups after the SARS outbreak at the beginning of the twenty-first century. In a study with SARS survivors, $25 \%$ of individuals were found to have symptoms of post-traumatic stress and $15 \%$ had depressive disorders approximately 3 years after the outbreak (7). Being in long-term quarantine and being a healthcare worker have been found to be associated with post-traumatic stress symptoms and feeling more psychological stress (8). Twenty per cent of those who contracted SARS were healthcare professionals, that was the highest rate of SARS infection than in any other group. In a study with healthcare workers 3 years after the outbreak, being younger, working in high-risk areas, being quarantined during an epidemic, and having family members-friends who contracted SARS were found to be associated with higher symptoms of post-traumatic stress, as well as the persistence of such symptoms in the long term (9). On the other hand, many other studies have revealed that altruistic attitudes in healthcare workers reduced negative psychological responses and depression symptoms. Having a higher level of education, being an experienced healthcare worker, and being adequately trained and supported for outbreaks have also been found to reduce the incidence of psychiatric illness in healthcare workers (10).

The effect of outbreaks on populations not directly affected by the disease, has rarely been a research topic investigated. In a population-based study conducted in Taiwan right after the SARS epidemic, $9 \%$ of the participants were found to be more pessimistic after the epidemic, and 11\% of them had psychiatric diseases such as sleep disorder, anxiety and depression. In this group, being over 50 years old, being highly educated and having concerns that SARS will recur were shown to be factors predicting symptomatic cases (11). In another study conducted with people who applied to community health centers, it was detected that $23 \%$ of 415 people had psychiatric problems related to SARS, especially symptoms of anxiety and depression, and $26 \%$ of them had posttraumatic stress symptoms. These rates were close to, or even higher than the rates found in previous studies with healthcare professionals or people surviving the disease (12) and it has been suggested that this situation may be related to the sense of uncertainty, lack of information and loss of control in the society. After the Middle East Respiratory Syndrome (MERS) epidemic in 2015 in Korea, in a study conducted with people who had contact with MERS patients and isolated due to the risk of infection, anxiety symptoms were detected in $7.6 \%$ and the feelings of anger in $16.6 \%$. The presence of anxiety and anger feelings after isolation was found to be associated with inadequate supply of daily necessities like food, water and self-care products, social activity limitation, history of psychiatric illness and financial loss (13).

Although COVID-19 is a new disease with yet unknown effects, studies have already started aiming to investigate its effects on mental health. In a study carried out in the Southwestern region of China in the early period of the COVID-19 epidemic, the prevalence of anxiety and depression were found higher in the group affected by quarantine $(12.9 \%$ 
and $22.4 \%$, respectively) than the group unaffected (6.7\% and $11.9 \%$, respectively). In the same study, it was also found that lower household income, lower education level, perception of one's general health as poor, lack of psychological support, and high level of economic loss due to pandemic were associated with anxiety and depression scores (14).

It is common for the society to experience fear in the first encounter with diseases of unknown origin and with probable fatal consequences. The individuals who were isolated during the MERS epidemic were afraid of being stigmatized and excluded by their neighbors alongside other psychological problems they experienced (13). Fear and lack of information or being misinformed often bring about stigmatizing and discrimination. During the SARS epidemic in United States, fear, stigmatization and discrimination reactions emerged against Asian people (15). When infection control techniques such as quarantine and isolation are implemented on affected groups, fear seems to rise up even more, and discriminatory behavior also increases considerably.

\section{Effects of epidemics on mental health of the elderly}

Studies investigating the effects of epidemics on elderly individuals have been carried out mainly on medical issues such as symptomatology, clinical course and treatment of the infection. The scarcity of studies examining how diseases and preventive measures affect the mental health of older adults is surprising. Elderly individuals are bound to be a heterogeneous group from different perspectives: they may be classified by age periods (younger aged (65-74 years), elderly (75-84 years), and older aged ones (85 and over), both physically and cognitively tend to be distinctive from each other), and by living conditions such as those living alone or in an extended family, retired or actively working, appearing to be unemployed but actively taking on responsibilities such as housework and caring for grandchildren, living independently or dependent on all daily activities, those with a permanent caregiver at home or in nursing homes. Thus the elderly are composed of many groups that differ from each other in terms of their financial and social opportunities. All these individuals with differing characteristics are anticipated to be affected by the epidemic differently on physical, economic and psychological grounds. Prior to the effects caused by the disease, prevention measures have important impacts on the mental health of older individuals.

SARS Cov-2 infection is known to be transmitted through droplets, for this reason increasing the social distance is the primary one among the measures taken for protection. In addition to the suggestions for the community-dwelling elderly to stay at home, not to enter the crowds or even reduce contact with family members, it is widely recommended to take measures to reduce the contact between caregivers, visitors and residents in hospitals and nursing homes, however all these measures also play a role in increasing social isolation.

Social isolation is known to increase the risk of cerebrovascular disease and cognitive impairment in the elderly and acts as an important risk factor on mortality (16). Numerous studies have reported that elderly individuals are significantly affected by loneliness and social isolation in their daily lives, and such isolation and lack of social support are associated with depression and suicide in elderly individuals (17). Social isolation has also been revealed to reduce the anti-viral immune response in older individuals (18). In addition to physical factors such as reduced immune response due to old age and accompanying chronic diseases, loneliness and isolation may increase the susceptibility of elderly individuals to various infectious diseases and COVID-19 through effects mentioned above.

The effect of outbreaks on mental health of the elderly is not limited to social isolation. Anxiety about getting sick and being a burden on the family, lack of support, limited access to healthcare system may also affect older individuals mentally. As a matter of fact, in a study conducted after the end of the SARS epidemic that caused the death 
of 299 people in Hong Kong between 1 November 2002 and 7 August 2003, suicide rates during the outbreak were found to be increased by $31 \%$ among the elderly individuals in Hong Kong compared to the same period of 2002 (19). The main increase in suicide rates was in elderly women, enhanced hopelessness associated with inability to access previous social support networks and healthcare services was considered to lead to the increase in suicidal behavior. In a retrospective analysis, suicide rates among elderly individuals in Hong Kong were found to be still high in 2004 (20). Examining the forensic records such as notes and suicide letters left by the individuals, some cases were revealed to be associated with the fear of getting sick, fear and anxiety associated with social isolation, and the fear of becoming a burden to the family (21). Elderly people with mental illness or cognitive disorders such as dementia are expected to experience exacerbation of mental and behavioral problems due to both exposure to stress and a possible infection during the epidemic; in addition, the infected elderly present frequently with delirium rather than the classical symptoms.

Case fatality rate for individuals over 60 years of age for SARS has been reported as $43.3 \%$. During the SARS epidemic, finding out that $70 \%$ of elderly people living in nursing homes in Hong Kong contracted the disease from the hospital, the visits to the hospitals were restricted during the epidemic period as a measure to prevent new infections. Elderly people with chronic illness and psychiatric disorders were adversely affected by this regulation, being unable to attend to regular followups and having difficulty in accessing treatment and their medications (22). The COVID-19 outbreak is likewise expected to increase inequality in access to healthcare in low-income countries and further marginalize elderly people who already have limited access to healthcare (23).

During SARS epidemic, outreach healthcare services offered to the residential homes and domiciliary home visits to the elderly patients were suspended due to the shortage of healthcare professionals (22). Dementia patients staying in longterm institutions and patients in geropsychiatric services have problems in following the infection control measures due to their cognitive problems; this fact increases the infection risk of healthcare personnel and other staff who are responsible for care, and the healthcare staff may be reluctant to take responsibility for the care and treatment of such patients, or take strict measures and reduce contact with the patients (22). Quarantine of nursing home staff who gets infected or who has contact with infected patients results in a shortage of care personnel in nursing homes, and overloading of the remaining staff leads to flaws in the care process. The nursing home residents may feel abandoned or lonely; depression, weight loss and behavioral problems may emerge in patients with dementia when further negative factors are added such as reduced human contact and warmth, living spaces arranged like a home resembling a hospital due to protective equipment, visitors banned, residents not allowed to gather in common areas and perform group activities, and being isolated in their rooms (24). The banning of the family visits may prevent the supervision of the service quality, especially in nursing homes operated by the private sector, and may create extra risk for such residents.

Another situation that negatively affects older individuals is the negative opinion that the elderly will pose an extra risk to healthcare professionals as "invisible SARS patients" due to the atypical clinical appearance of the disease in the elderly. Furthermore, there is a high risk of labelling the older people as "hopeless cases", "useless, unproductive" people, and regarding them as a burden on the system (22). As a matter of fact, both negative and stigmatizing posts about elderly people, negative stereotypes about age and "ageism" became more uttered on social media in both SARS epidemic and COVID-19 pandemic (25).

Shortage in staff and resources that may be encountered in the healthcare system during the 
epidemic will also bring about ethical problems: during the COVID-19 outbreak, setting an age limit for the admission to intensive care, limiting the admission of the elderly with lesser years of survival or with a diagnosis of dementia to the hospital, instead saving the scarce sources for younger and healthy people have been frequently debated (26). In addition to being aware of the possibility of such a discrimination, fear of getting sick and dying would also trigger symptoms of anxiety and depression in elderly, yet increase the possibility of domestic violence and neglect.

It has been several months since the outbreak of the COVID-19 pandemic, and despite the now wellknown fact that the viral outbreaks affect the mental health of older people from previous experiences, the scarcity of studies examining the mental health of elderly individuals is rather striking. In a survey conducted among elderly people in China, 37\% of the people over the age of 60 were found to experience depression or anxiety, and women were found to be more affected than men (27). On the other hand, in some studies older people were found to experience lower anxiety and depression symptoms compared to younger ones (14). This finding is explained as those who live into the eighth or ninth decade of their lives in the present time have already survived many difficult periods such as the Second World War and economic crises, and gained resistance to stress. However more studies are needed to reach a definite conclusion.

\section{Conclusions and recommendations}

It seems that epidemics or pandemics associated with viruses will likely continue to pose a threat to humanity for a long time, with the contribution of globalization increasing the contraction risk (28). Governments, health authorities, public institutions and individuals need to be prepared for viral outbreaks in the future. Pre-drawn road maps will play a significant role in reducing contraction, mortality and morbidity in similar outbreaks in the future. Outbreaks affect the mental health as well as the physical health. Outbreaks and also the infection control measures affect the mental health of elderly people who has a high risk of morbidity and mortality, and similar groups such as the healthcare workers, infected people and their close contacts and the poor with a limited access to healthcare services. Some short- and long-term measures could be implemented to protect the mental health of older people in present and future epidemics.

Initially all healthcare professionals, such as family physicians, nurses, physiotherapists, and especially mental health professionals working with elderly individuals should all be informed about the psychological reactions that may be seen in the elderly during the epidemics; they should evaluate the suicidal thoughts, anxiety and depression symptoms in all elderly patients they encounter. Large-scale studies examining the effects of outbreaks on the mental health of older individuals should be planned and conducted respectively. In addition to the research examining the factors predisposing to stress and negative psychological responses, widespread studies should be encouraged to investigate the factors predicting resilience in different groups. While planning protection measures for elderly people, collaboration of local and general administrations, health authorities, professional organizations and the participation of relevant non-governmental organizations and community representatives in decision-making mechanisms would provide more effective results. Maintaining social distance should be the most important and primary method in elderly individuals as in all vulnerable groups, but all measures to be taken for assuring the distance should be arranged so as not to increase the sense of loneliness and isolation. In today's world, social media and the internet are highly used modalities for the public information; but considering the limited use of the internet and social media by elderly, they should be informed through traditional media like television and newspapers. Local announcements through local authorities such 
as municipalities and mukhtars in our country, and even announcements by religious services could also be used in appropriate situations. The suspension of routine healthcare services during epidemics may cause the elderly to feel helpless and abandoned; for this reason, "clean" healthcare services apart from pandemic hospitals should be created for the elderly to continue their routine healthcare and follow-up of the chronic conditions. Online counseling and online mental health services have become widespread in the 21 st century and have also been widely used during epidemics. Considering the majority of elderly individuals not being familiar with internet services, more traditional methods such as telephone counseling/ hotlines should be provided for this group. Mental health support lines providing services only for the elderly could be established, and voluntary mental health workers could reach elderly individuals who live alone through the support lines.

Through non-governmental organizations, volunteers could reach out for help to the elderly

\section{REFERENCES}

1. https://www.who.int/docs/default-source/ coronaviruse/situation-reports/20200622-covid-19sitrep-154.pdf?sfvrsn=d0249d8d_2. Accessed: 23. 06. 2020

2. https://www.who.int/publications/i/item/modes-oftransmission-of-virus-causing-covid-19-implicationsfor-ipc-precaution-recommendations

3. Liu Y, Mao B, Liang $S$ et al. Association between age and clinical characteristics and outcomes of COVID-19. Eur Respir J 2020; 55 (5): 2001112. (PMID: 32312864)

4. Lauretani F, Ravazzoni G, Roberti MF et al. Assessment and treatment of older individuals with COVID-19 multi-system disease: clinical and ethical implications. Acta Biomed 2020; 91 (2): 150-168. (PMID: 32420939)

5. Lloyd-Sherlock $\mathrm{P}$, Kalache $\mathrm{A}, \mathrm{McKee} \mathrm{M}$ et al. WHO must prioritise the needs of older people in its response to the COVID-19 pandemic. BMJ 2020; 31, 368: m1164. (PMID: 32205399) in various ways; these services may include regular contact with elderly people over the phone and helping for the daily needs of those living alone. The establishment of coordination systems for the execution and control of such activities could be planned. Measures should be taken to protect older individuals residing in elderly care centers and nursing homes, but also arrangements should be made to gather them with their relatives in a controlled manner in order to reduce the feelings of loneliness, abandonment, and avoid the lack of stimulus and related behavioral problems. For taking early measures to prevent negative psychological consequences in the current and future outbreaks, relevant studies should be carried out about the psychosocial problems and mental health needs of the elderly.

6. Torales J, O'Higgins M, Castaldelli-Maia JM et al. The outbreak of COVID-19 coronavirus and its impact on global mental health. Int J Soc Psychiatry 2020; 66 (4): 317-320. (PMID: 32233719)

7. Mak IWC, Chu CM, Pan PC et al. Long-term psychiatric morbidities among SARS survivors. Gen Hospital Psych 2009; 31 (4): 318-26. (PMID: 19555791)

8. Reynolds DL, Garay JR, Deamond SL et al. Understanding, compliance and psychological impact of the SARS quarantine experience. Epidemiol Infect 2008; 136 (7): 997-1007. (PMID: 17662167)

9. Wu P, Fang Y, Guan Z et al. The psychological impact of the SARS epidemic on hospital employees in China: exposure, risk perception, and altruistic acceptance of risk. Can J Psychiatry 2009; 54 (5): 302-311. (PMID: 19497162)

10. Lancee WJ, Maunder RG, Goldbloom DS et al. Prevalence of psychiatric disorders among Toronto hospital workers one to two years after the SARS outbreak. Psychiatr Serv 2008; 59 (1): 91-95. (PMID: 18182545) 
11. Peng EY, Lee MB, Tsai ST et al. Population-based post-crisis psychological distress: an example from the SARS outbreak in Taiwan. J Formos Med Assoc 2010; 109 (7): 524-532. (PMID: 20654792)

12. Sim K, Chan YH, Chong PN et al. Psychosocial and coping responses within the community health care setting towards a national outbreak of an infectious disease. J Psychosom Res 2010; 68 (2): 195-202. (PMID: 20105703)

13. Jeong $\mathrm{H}$, Yim HW, Song YJ et al. Mental health status of people isolated due to Middle East Respiratory Syndrome. Epidemiol Health 2016; 38: e2016048. (PMID: 28196409)

14. Lei $L$, Huang $X$, Zhang $S$ et al. Comparison of prevalence and associated factors of anxiety and depression among people affected by versus people unaffected by quarantine during the COVID-19 epidemic in Southwestern China. Med Sci Monit 2020; 26: e924609. (PMID: 32335579)

15. Person B, Sy F, Govert B et al. Fear and stigma: the epidemic within the SARS outbreak. Emerg Infect Dis 2004; 10 (2): 358-362. (PMID: 15030713)

16. Friedler B, Crapser J, McCullough L. One is the deadliest number: the detrimental effects of social isolation on cerebrovascular diseases and cognition. Acta Neuropathol 2015; 129 (4): 493-509. (PMID: 25537401)

17. Conwell $Y$, Duberstein PR, Caine ED. Risk factors for suicide in later life. Biol Psychiatry 2002; 52: 193-204. (PMID: 12182926)

18. Cole SW, Hawkley LC, Arevalo JM et al. Social regulation of gene expression in human leukocytes. Genome Biol 2007; 8 (9): R189. (PMID: 17854483)

19. Chan SM, Chiu FK, Lam LCW et al. Elderly suicide and the 2003 SARS epidemic in Hong Kong. Int J Geriatr Psychiatry 2006; 21 (2): 113-118. (PMID: 16416469)

20. Cheung YT, Chau PH, Yip PS. A revisit on older adults' suicides and severe acute respiratory syndrome (SARS) epidemic in Hong Kong. Int J Geriatr Psychiatry 2008; 23 (12): 1231-1238. (PMID: 18500689)

21. Yip PS, Cheung YT, Chau PH et al. The impact of epidemic outbreak: the case of severe acute respiratory syndrome (SARS) and suicide among older adults in Hong Kong. Crisis 2010; 31 (2): 86-92. (PMID: 20418214)

22. Chiu HF, Lam LC, Li SW et al. SARS and psychogeriatrics: perspective and lessons from Hong Kong. Int J Geriatr Psychiatry 2003; 18 (10): 871-873. (PMID: 14533118)
23. Lloyd-Sherlock $P$, Ebrahim $S$, Geffen $L$ et al. Bearing the brunt of COVID-19: older people in low and middle income countries. BMJ 2020; 368: m1052. (PMID: 32169830)

24. Gardner W, States D, Bagley N. The coronavirus and the risks to the elderly in long-term care. J Aging Soc Policiy 2020; 3: 1-6. PMID: 32245346; Fallon A, Dukelow T, Kennelly SP et al. COVID-19 in nursing homes. QJM 2020; 113 (6): 391-392. (PMID: 32311049)

25. Jimenez-Sotomayor MR, Gomez-Moreno C, SotoPerez-de-Celis E. Coronavirus, ageism, and twitter: an evaluation of tweets about older adults and COVID-19. J Am Geriatr Soc 2020; 27: 10.1111/ jgs.16508. (PMID: 32338787)

26. Cesari M, Proietti M. COVID-19 in Italy: ageism and decision-making in a pandemic. J Am Med Dir Assoc 2020; 21 (5): 576-577. (PMID: 32334771)

27. Meng H, Xu Y; Dai J et al. Analyze the psychological impact of COVID-19 among the elderly population in China and make corresponding suggestions. Psychiatry Res 2020; 289: 112983. (PMID: 32388175)

28. Wei P, Cai Z, Hua J et al. Pains and gains from China's experiences with emerging epidemics: from SARS to H7N9. Biomed Res Int 2016; 2016: 5717108. (PMID: 27525272) 